\title{
Combined use of peptide receptor radionuclide therapy and metronomic chemotherapy in neuroendocrine tumors: a possible choice driven by nuclear medicine molecular imaging
}

\author{
Fabio Minutoli ${ }^{1,4}\left(\mathbb{D} \cdot\right.$ Riccardo Laudicella $^{1,2}\left(\mathbb{D} \cdot\right.$ Irene A. Burger $^{2,3}(\mathbb{D}) \cdot$ Sergio Baldari ${ }^{1,4} \mathbb{C}$ \\ Published online: 13 April 2021 \\ (C) The Author(s), under exclusive licence to Springer-Verlag GmbH Germany, part of Springer Nature 2021
}

Dear Sir,

Neuroendocrine tumors (NETs) are relatively rare tumors mainly originating from gastro-entero-pancreatic (GEP) areas and can produce bioactive amines and hormones. NETs are often slow-growing and if non-secretive usually asymptomatic and diagnosed in a metastatic stage. GEP NETs are pathologically classified according to their Ki67 proliferation index or mitotic rate [1]. Pathological characterization of GEP NETs is mandatory to confirm the diagnosis, recognize grade, and select the appropriate therapy. Treatment is multidisciplinary and should be individualized according to the tumor type, burden, and symptoms. Therapeutic tools include surgery, interventional radiology, medical treatments such as somatostatin analogues, interferon, chemotherapy, targeted drugs (everolimus, sunitinib), and radiolabelled somatostatin analogues or particles for selective internal radiotherapy. Peptide receptor radionuclide therapy (PRRT) using radiolabelled somatostatin analogues has been used for more than 20 years in an increasing number of centers, mainly in GEP NETs with high somatostatin receptor II (SSTR) expression. Dosimetric studies demonstrated that $\left[{ }^{90} \mathrm{Y}\right]$ - and $\left[{ }^{177} \mathrm{Lu}\right] \mathrm{DOTA}$-conjugates can deliver high radiation doses to SSTR expressing tumors and

This article is part of the Topical Collection on Oncology - Digestive Tract.

Sergio Baldari

sbaldari@unime.it

1 Department of Biomedical and Dental Sciences and Morpho-Functional Imaging, University of Messina, Messina, Italy

2 Department of Nuclear Medicine, University Hospital Zürich, University of Zürich, Zürich, Switzerland

3 Department of Nuclear Medicine, Kantonsspital Baden, Baden, Switzerland

4 Nuclear Medicine Unit, University Hospital "Policlinico G. Martino", Via Consolare Valeria 1, 98125 Messina, Italy low doses to normal organs. With the NETTER trial, a prospective randomized study, it could show that both the progression free (PFS) and overall survival is significantly higher for $\left[{ }^{177} \mathrm{Lu}\right]$ DOTATATE compared with long-acting octreotide with a PFS of 65.2 vs. $10.8 \%$ at 20 months [2]. Side effects, mainly involving kidney and bone marrow, are limited in incidence and usually transient and mild, especially for $\left[{ }^{177} \mathrm{Lu}\right]$-labeled peptides. Renal protection is used to further minimize the risk of a late decrease in renal function [3]. Therefore, recently EMA approved $\left[{ }^{177} \mathrm{Lu}\right]$ oxodotreotide (®Lutathera) for the treatment of adult patients harboring SSTR-positive, progressive, non-removable, or metastatic well-differentiated (G1 and G2) GEP-NETs. Nevertheless, NETs are rather heterogeneous tumors [4], ranging from slow-growing tumors highly expressing SSTRs to aggressive lesions with scant or absent SSTR expression. This heterogeneity may characterize different lesions in the same patient, the same lesions at different time points, or even both. One focal biopsy is therefore unable to map and fully depict the tumor heterogeneity. Nuclear Medicine imaging can overcome such limitations: when clinically required, $\left[{ }^{18} \mathrm{~F}\right] \mathrm{FDG}$ PET information may integrate SSTR imaging. Based on their glucose uptake, $\left[{ }^{18} \mathrm{~F}\right] \mathrm{FDG}$ PET can reveal aggressiveness of lesions [5] and provide prognostic information [6-8]. Interestingly, prognostic stratification based on FDG PET classifies patients better than pathology [8] and integration with SSTR imaging data may provide an accurate evaluation of biological characteristics of lesions along a continuum from SSTR-positive and FDG-negative lesions to SSTR-negative and FDG-positive lesions [9].

The study by Nicolini et al. highlights the capability of nuclear medicine imaging using both $\left[{ }^{18} \mathrm{~F}\right] \mathrm{FDG}$ PET and radiolabeled somatostatin analogs imaging to drive therapeutic choices in GEP NET patients, representing a great example for stratification of patients based on molecular imaging [10]. They analyzed combined metronomic chemotherapy-PRRT treatment, using $\left[{ }^{177} \mathrm{Lu}\right]$ DOTATATE, in metastatic patients 
with widely different pathological grading (G1-G3, Ki67< $55 \%$ ) in combination with capecitabine (pro-drug of 5-fluorouracil) [10]. Key for patient inclusion was to select subjects showing lesions with a high uptake on SSTR PET (grade 3 or 4 according to Krenning score) and at least one documented lesion positive on FDG PET (with SUV max >2.5). These rigorous inclusion criteria are the strength of the study, since they allow testing this combined therapy in patients showing characteristics that suggest limited effectiveness of PRRT alone. The rationale to apply the use of a radiosensitizer in patients with an expected poorer prognosis is to enhance the biological effect of relatively penetrating beta negative particles on metabolically active malignant cells by increasing the likelihood of cell death due to the added damage caused by capecitabine. Compared with previous studies evaluating PRRT alone in patients with FDG positive NET tumors, the results of Nicolini et al. showed an improved PFS with a similar performance in terms of disease control rate and side effects. In fact, toxicity was mainly mild and transient, and only a limited percentage of patients had to discontinue the combined $\left[{ }^{177} \mathrm{Lu}\right]$ DOTATATE-metronomic capecitabine treatment. This supports the rationale to use radiosensitizers to improve PRRT efficacy in a subgroup of patients identified by molecular imaging. This positive phase II study and previous results in the field $[10,11]$ support the need for randomized comparative studies.

Some clinical trials combining PRRT and the use of capecitabine, 5-fluorouracil, temozolomide, or everolimus with different schedules are growing worldwide and supportive results are expected from the Scientific Community; moreover, new therapeutic approaches are being tested in a preclinical setting [12-14]. Many open issues remain in the treatment of NETs, such as best sequencing of different treatments, use of combined treatments and their schedule, best PRRT radiopharmaceutical, personalized dosimetric evaluation versus standard dosing, therapy response assessment, artificial intelligence application, and so on. In our opinion, the paramount issue is still the need for improved stratification of patients in terms of prognosis and biological characteristics of their disease, aiming to select the best fitting approach with the best efficacy and reduced toxicity and cost (precision medicine). Standardization of patients' stratification mainly based on biological characteristics revealed by molecular imaging should represent a pivotal clinical instrument in addressing therapies. In this field, NETs represent the paradigm for the successful use of nuclear medicine theragnostics towards personalized therapies.

\section{Declarations}

Ethical approval This article does not contain any study with human participants or animals performed by the author.
Conflict of interest The authors declare that they have no conflict of interest.

\section{References}

1. Nagtegaal ID, Odze RD, Klimstra D, Paradis V, Rugge M, Schirmacher P, et al. WHO classification of Tumours editorial board. The 2019 WHO classification of tumours of the digestive system. Histopathology. 2020;76:182-8.

2. Strosberg J, El-Haddad G, Wolin E, Hendifar A, Yao J, Chasen B, et al. Phase 3 trial of (177)Lu-Dotatate for Midgut neuroendocrine tumors. N Engl J Med. 2017;376:125-35.

3. Severi S, Grassi I, Nicolini S, Sansovini M, Bongiovanni A, Paganelli G. Peptide receptor radionuclide therapy in the management of gastrointestinal neuroendocrine tumors: efficacy profile, safety, and quality of life. Onco Targets Ther. 2017;10:551-7.

4. Modlin IM, Oberg K, Chung DC, Jensen RT, de Herder WW, Thakker RV, et al. Gastroenteropancreatic neuroendocrine tumours. Lancet Oncol. 2008;9:61-72.

5. Pencharz D, Gnanasegaran G, Navalkissoor S. Theranostics in neuroendocrine tumours: somatostatin receptor imaging and therapy. Br J Radiol. 2018;91:20180108.

6. Severi S, Nanni O, Bodei L, Sansovini M, Ianniello A, Nicoletti S, et al. Role of 18FDG PET/CT in patients treated with $177 \mathrm{Lu}-$ DOTATATE for advanced differentiated neuroendocrine tumours. Eur J Nucl Med Mol Imaging. 2013;40:881-8.

7. Bahri H, Laurence L, Edeline J, Leghzali H, Devillers A, Raoul JL, et al. High prognostic value of 18 F-FDG PET for metastatic gastroenteropancreatic neuroendocrine tumors: a long-term evaluation. J Nucl Med. 2014;55:1786-90.

8. Binderup T, Knigge U, Loft A, Federspiel B, Kjaer A. 18Ffluorodeoxyglucose positron emission tomography predicts survival of patients with neuroendocrine tumors. Clin Cancer Res. 2010;16:978-85.

9. Chan DL, Pavlakis N, Schembri GP, Bernard EJ, Hsiao E, Hayes A, et al. Dual somatostatin receptor/FDG PET/CT imaging in metastatic neuroendocrine tumours: proposal for a novel grading scheme with prognostic significance. Theranostics. 2017;7:1149-58.

10. Nicolini S, Bodei L, Bongiovanni A, Sansovini M, Grassi I, Ibrahim T, et al. Combined use of 177Lu-DOTATATE and metronomic capecitabine (Lu-X) in FDG-positive gastro-entero-pancreatic neuroendocrine tumors. Eur J Nucl Med Mol Imaging. 2021. https://doi.org/10.1007/ s00259-021-05236-z Online ahead of print.

11. Kashyap R, Hofman MS, Michael M, Kong G, Akhurst T, Eu P, et al. Favourable outcomes of (177)Lu-octreotate peptide receptor chemoradionuclide therapy in patients with FDG-avid neuroendocrine tumours. Eur J Nucl Med Mol Imaging. 2015;42:176-85.

12. Nonnekens J, van Kranenburg M, Beerens CE, Suker M, Doukas $\mathrm{M}$, van Eijck $\mathrm{CH}$, et al. Potentiation of peptide receptor radionuclide therapy by the PARP inhibitor Olaparib. Theranostics. 2016;6:1821-32.

13. Lundsten S, Spiegelberg D, Stenerlöw B, Nestor M. The HSP90 inhibitor onalespib potentiates $177 \mathrm{Lu}$-DOTATATE therapy in neuroendocrine tumor cells. Int J Oncol. 2019;55:1287-95.

14. Ognjanović M, Radović M, Mirković M, Prijović Ž, Puerto Morales MD, Čeh M, et al. 99mTc-, 90Y-, and 177Lu-labeled Iron oxide Nanoflowers designed for potential use in dual magnetic hyperthermia/radionuclide Cancer therapy and diagnosis. ACS Appl Mater Interfaces. 2019;11:41109-17.

Publisher's note Springer Nature remains neutral with regard to jurisdictional claims in published maps and institutional affiliations. 